MATEC Web of Conferences 16, 03005 (2014)

DOI: $10.1051 /$ matecconf/ 20141603005

C Owned by the authors, published by EDP Sciences, 2014

\title{
Method of the unbalanced rotor critical frequency overcoming by means of the operated magnetic spring
}

\author{
Alexey Lukin ${ }^{1}$, Ivan Popov ${ }^{1}$, Dmitry Skubov ${ }^{1,2, a}$ and Lev Shtukin ${ }^{1,2}$ \\ ${ }^{1}$ St. Petersburg State Polytechnic University, Mechanics and Control Processes Department \\ ${ }^{2}$ Institute of Problems of Mechanical EngineeringRAS
}

\begin{abstract}
When passing critical rotational frequency of a rotor with significant imbalance there can be crossoscillations with large amplitudes. The algorithm of critical rotational frequency overcoming which allows reducing these oscillations considerably is described.It is offered to use a differential magnetic spring as an elastic support with operated stiffness.
\end{abstract}

When passing critical rotational frequency of a rotor with significant imbalance there can be cross-oscillations with large amplitudes. The algorithm of critical rotational frequency overcoming which allows reducing these oscillations considerably is offered. For this aim it's proposed that elastic supports have two stiffness coefficients: working and increased, with the corresponding two values of critical frequency. It is offered to use a differential magnetic spring as an elastic support with operated stiffness. The magnetic spring consists of the permanent magnets connected by magnetic conductors. The schematic diagram of a differential magnetic spring is shown in Fig. 1.

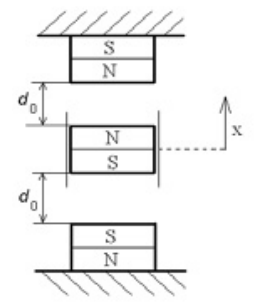

Fig. 1 - The scheme of the magnetic spring consisting of three permanent magnets

Its important advantage is capability to change easily the equivalent stiffness of an elastic support. It allows to operate the value of stiffness during the rotor acceleration so that to achieve reduction of oscillations amplitude, and, therefore, stresses in the supports up to the acceptable values even at big imbalance and to provide the acceleration to the over-critical frequency of rotation. Technically it is quite possible to change (to reduce) stiffness of a magnetic spring (to increase an initial gap) quickly. If to make fastening of magnets by means of a latch, atits release magnets can be moved apart under the force of their interaction, thereby increasing an initial gap for rather small time.
The magnetic support (spring) contains three permanent magnets, side magnets are fixed and the middle one, which is connected with the shaft train, can move in the vertical direction, and is itself established on the amortized support. Dependences of force $F(x)$ acting on a middle permanent magnet, on vertical displacement $x$ at various values of an initial gap $d_{0}$ are given in Fig. 2 . These dependences are received by analysis of a stationary magnetic field in system of magnets with the subsequent calculation of force of their interaction.

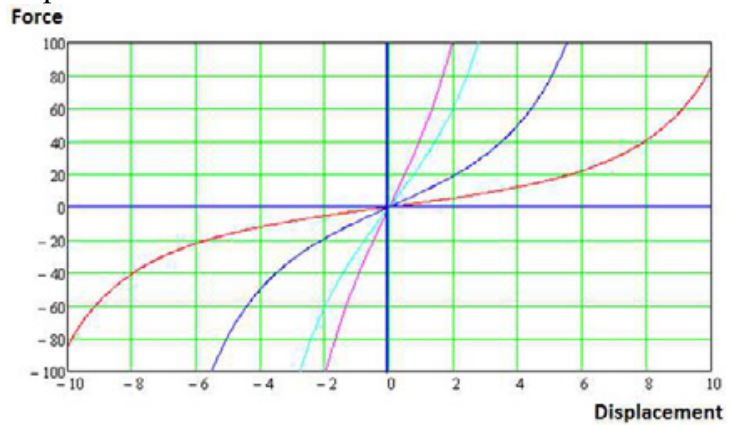

Fig.2 -Stiffness characteristic of a magnetic spring

Varying the size of an initial gap between extreme magnets, it is possible to change the size of equivalent stiffness (Fig. 2), thereby changing a skeletal curve. The established oscillations of a platform at the fixed position of magnets and, therefore, amplitude-frequency characteristics can be found by solving the shaft train dynamics equations by a harmonic balance method. In Fig. 3 dependence of a platform oscillations amplitude on the rotation frequency is shown at various values of an initial gap in a magnetic spring (amplitude-frequency characteristics).

$\overline{{ }^{a} \text { Corresponding author: skubov.dsk } @ y a n d e x . r u ~}$ 


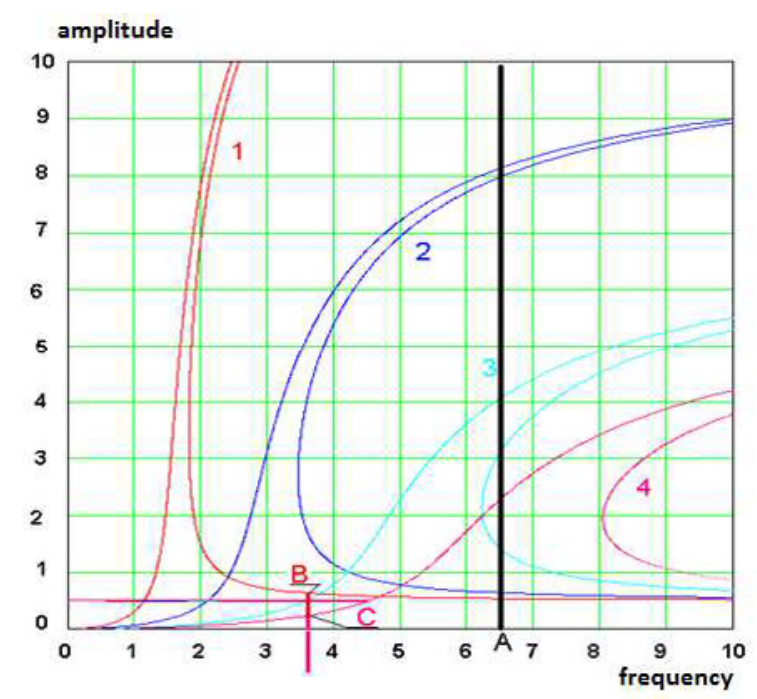

Fig.3 - Amplitude-frequencycharacteristics

The essence of offered algorithm of operated transition through a resonance consists in the following. Let the frequency at A point correspond to an operating mode, and the support stiffness at an operating mode correspond to the characteristic No.1 (Fig.3). At such support stiffness it is inevitably to overcome a resonant zone with big amplitudes of oscillations. Before acceleration of the rotor the increased stiffness of supports turns on. At the first stage of acceleration the rotor reaches the frequency exceeding the working critical frequency, but not exceeding critical frequency, which corresponds to the increased stiffness (see characteristic No.4). Then working stiffness joins, and acceleration to the working frequency of rotation proceeds. Thereby throughout all rotor acceleration process the frequency of rotation isn't close to actual critical frequency.

When designing such a support a number of questions arise. In particular, how fast the process of stiffness change should be carried out, as at slow change of a gap the mode of oscillations will be almost established, thus it will correspond to the top branch of characteristic No.4, and it won't turn out to get on the bottom branch (point B). Numerical and analytical researches are necessary for the analysis of such nonlinear dynamic system, as a result of which its characteristics corresponding to various criteria such as the minimum time of transition to the operating frequency or the minimum of the oscillations amplitude, etc., should be received.

For research of an unbalanced rotor cross oscillations the nonlinear non-uniform differential equation with one degree of freedom is considered. The accounting of only one (first) form of oscillations is proposed. Dimensionlessdynamicsequationhasthefollowingform:

$$
\ddot{x}+2 \varepsilon n \dot{x}+F(x, t)=e \Omega^{2} \cos (\Omega t),
$$

wheren - friction coefficient, $\Omega$ - the value of excitement frequency, $e-$ unbalance parameter.

Using the static characteristic of magnetic spring stiffness, we will accept it in the form of polynomial dependence with a time-dependent coefficient. In case of small speeds of a support stiffness change it is possible to write $F(x, t)=\left(1-e^{-\varepsilon t}\right)\left(\alpha x^{3}-\lambda \delta x\right)$. Thenequation (1) takestheform:

$$
\begin{aligned}
& \ddot{x}+2 \varepsilon n \dot{x}+\left(\lambda-\delta\left(1-e^{-\varepsilon t}\right)\right) x+ \\
& +\alpha\left(1-e^{-\varepsilon t}\right) x^{3}=e \Omega^{2} \cos (\Omega t) .
\end{aligned}
$$

where: $\lambda$ - the square of the free oscillations frequency at the initial magnets position, $(\lambda-\delta)-$ the square of the frequency after moving magnets apart. Parameter $\varepsilon$ characterizes the speed of stiffness change - the speed of drawing magnets apart. In relative time it correlates to a period of free oscillations.

Upon slow transition (time of transition makes some periods of cross oscillations), at a small deviation of excitement frequency from the resonant one and at low external friction the solution of the equation (2) is searched by an averaging method, also known as a projective method, in the form of the sum of harmonicas with slowly changing coefficients [4]:

$$
x(t)=z_{1}(\varepsilon t) \sin \Omega t+z_{2}(\varepsilon t) \cos \Omega t .
$$

As a result of application of a projective method we receive system of the differential equations for slowly varying coefficients:

$$
\left\{\begin{aligned}
\ddot{z}_{1}+ & 2 n \varepsilon \dot{z}_{1}+\left(\lambda-\delta\left(1-e^{-\varepsilon t}\right)\right) z_{1}-\Omega^{2} z_{1}-2 \Omega \dot{z}_{2}- \\
& -2 n \varepsilon \Omega z_{2}+\frac{3}{4} \alpha\left(1-e^{-\varepsilon t}\right)\left(z_{1}^{3}+z_{1} z_{2}^{2}\right)=0 \\
\ddot{z}_{2} & +2 n \varepsilon \dot{z}_{2}+\left(\lambda-\delta\left(1-e^{-\varepsilon t}\right)\right) z_{2}-\Omega^{2} z_{2}+2 \Omega \dot{z}_{1}+ \\
& +2 n \varepsilon \Omega z_{1}+\frac{3}{4} \alpha\left(1-e^{-\varepsilon t}\right)\left(z_{2}^{3}+z_{2} z_{1}^{2}\right)=e \Omega^{2}
\end{aligned}\right.
$$

where $\left(\lambda-\delta\left(1-e^{-\varepsilon t}\right)\right)$ - function of small oscillations eqigenfrequency change (reduction) at slow separation of magnets.

Comparison of the approximate solution of system (4) with the numerical solution of the initial equation (2) showed good compliance of results in the wide range of external frequency $\Omega$ values. The results for $\Omega=0,8$ and $1,2, \lambda=1, \delta=0.5$ are shown in Fig. 4,5 respectively.

A gradual divergence between solutions, which is theoretically expected for projective solutions of differential equations with slowly changing coefficients [3], was observed.

The characteristic matrix eigenvalues of linear part of system (4), which depend on "slow time" $\varepsilon t$, play an essential role in proximity of projective and direct numerical calculation of transitional oscillations. The best convergence of results of numerical and projective solutions requires the greatest possible increase in the module of negative real part of the eigenvalues. According to preliminary results, at some ratio of parameters it is possible that eigenvalues real parts have positive values.Futher researches are necessary here. 


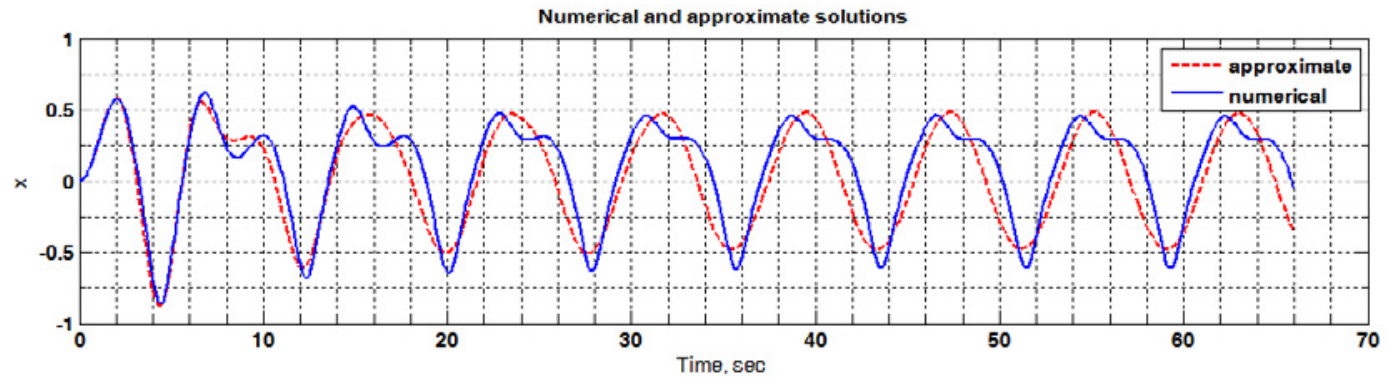

Fig.4-Comparison of the approximate and numerical solutions, $\Omega=0,8$

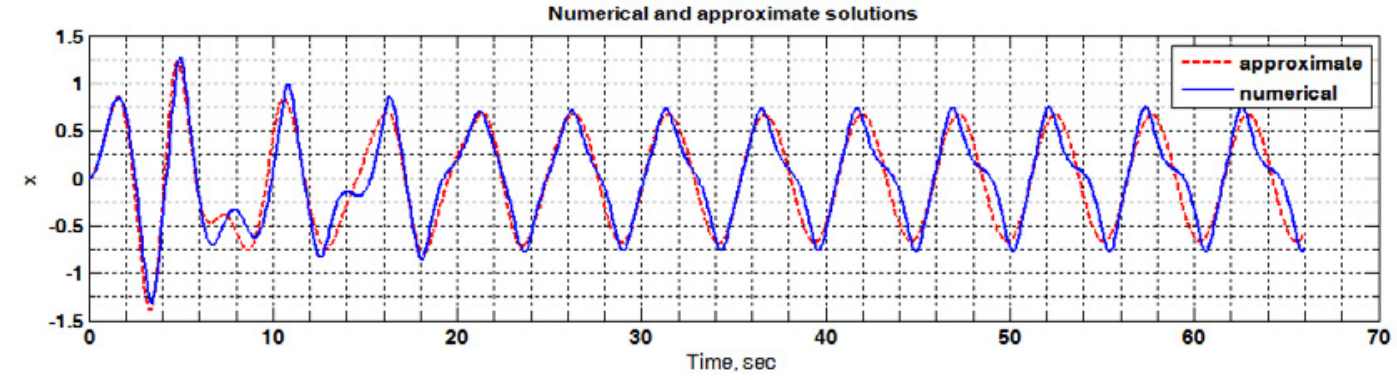

Fig. 5 - Comparison of the approximate and numerical solutions, $\Omega=1,2$

The described mathematical model (2) doesn't consider some features of a shaft critical frequency overcoming process. First, a stationary value of external excitement frequency is considered, while it makes the great interest to study the effects connected with change of frequency of rotation (excitement). Secondly, at application of a projective method it is supposed that parameter $\varepsilon$, corresponding in this system to the speed of a support stiffness change, is small that limits area of applicability of this method. In this regard, more detailed mathematical model of system was considered:

$$
\begin{aligned}
& \ddot{x}+2 \varepsilon_{d} \dot{x}+\left\{\lambda-\delta\left[1-\mathrm{e}^{\left(-H\left(t-t_{*}\right) \varepsilon_{t}\left(t-t_{*}\right)\right)}\right]\right\} x+ \\
& +\alpha\left[1-\mathrm{e}^{\left(-H\left(t-t_{*}\right) \varepsilon_{t}\left(t-t_{*}\right)\right)}\right] x^{3}= \\
& \varepsilon_{\Omega}\left(\Omega_{0}+\varepsilon_{t \Omega} t\right)^{2} \cos \left[\left(\Omega_{0}+\varepsilon_{t \Omega} t\right) t\right],
\end{aligned}
$$

where $\varepsilon_{d}$ - friction coefficient; $\varepsilon_{t}-$ parameter (not obligatory small) responsible for the speed of a support stiffness change; $t_{*^{-}}$timepoint in which change of a support stiffness begins; $H\left(t-t_{*}\right)-$ Heaviside step function; $\varepsilon_{\Omega}$ - external excitement amplitude; $\Omega_{0}$ - initial external excitement frequency; $\varepsilon_{t \Omega}-$ speed of external excitement frequency growth.

The equation (5) was solved numerically for various values of varied parameters - the speed, the moment and the value of stiffness change; system eigenfrequency and the speed of a rotor acceleration; the value of a cubic nonlinearity contribution.

In Fig. 6 the process of a shaft cross oscillations is shown in a case when working stiffness joins at the beginning of rotor acceleration. This case, in essence, is equivalent to acceleration to working frequency without a support stiffness change.

According to the results, in case of an unbalanced rotor acceleration the maximum amplitude of cross oscillations is reached not at the time of eigenfrequency and excitement frequency coincidence, but slightly earlier, i.e. the maximum of amplitude moves to the area of lower frequencies.

In this regard, the initial assumption that the frequency of external excitement at which there is a change of magnetic support stiffness should be in range $\left[\omega_{1}, \omega_{2}\right]$, where $\omega_{1}-$ the working cross oscillations eigenfrequency; $\omega_{2}-$ the increased eigenfrequency, was clarified. Namely, it was found out that optimum value $\Omega_{*}<\omega_{1}$.

In Fig 7,8 the process of a shaft cross oscillations is shown in a case when working stiffness joins at the moment $t_{*}$ when the excitement frequency makes $\Omega_{*}=0,4 \omega_{2} \quad$ (we will note that $\omega_{1}=0,5 \omega_{2}$ ).

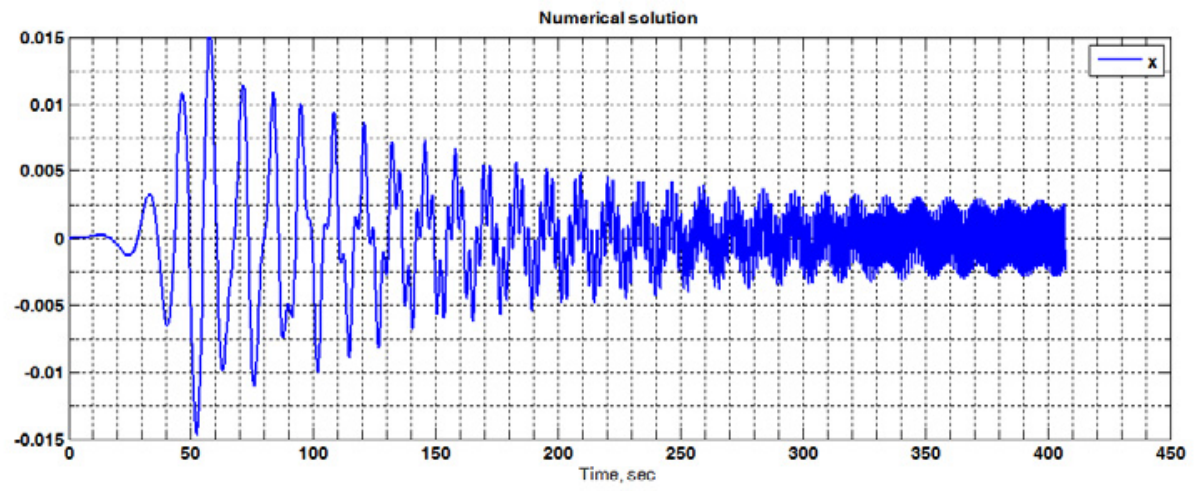

Fig.6 -Shaft cross oscillations (no support stiffness change)

$\overline{{ }^{a} \text { Corresponding author: skubov.dsk } @ y a n d e x . r u ~}$ 


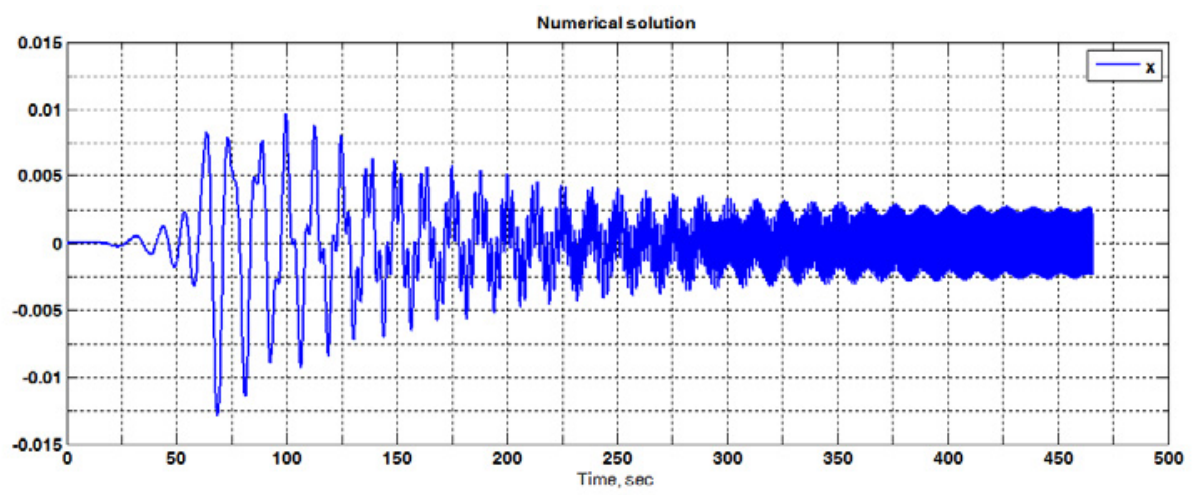

Fig. 7 - Shaft cross oscillations $\left(\Omega_{*}=0,4 \omega_{2}\right)$

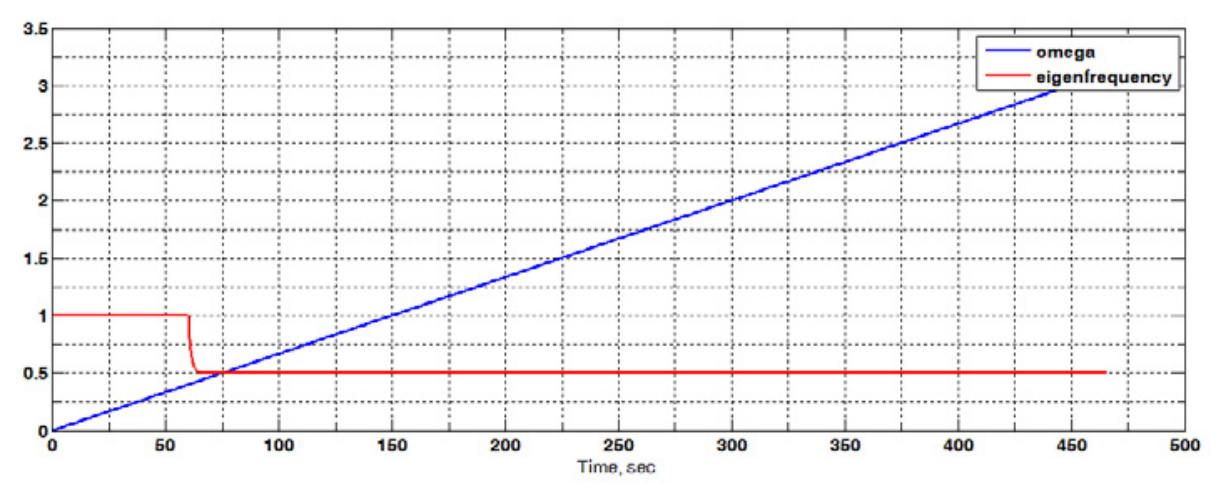

Fig. 8 - Eigenfrequency and excitement frequency dependence on time $\left(\Omega_{*}=0,4 \omega_{2}\right)$

We see that amplitude of resonant oscillations decreased by $30 \%$ in comparison with a case of acceleration to working frequency without support stiffness change.

It is necessary to define an optimum time point (optimum frequency of external excitement) in which support stiffness change should be performed. In Fig 9, 10 results of this research are presented at $\omega_{2}=1$ and $\omega_{2}=5$ respectively. In both cases $\omega_{1}=0,5 \omega_{2}$. X-axis represents the values of relative excitement frequency $\Omega_{*} / \omega_{2}, \mathrm{Y}$-axis - rezonant amplitude values, normalized to the value of resonant amplitude in a case of constant support stiffness during the whole process of acceleration. Results show that stiffness change at an optimum frequency $\Omega_{*} \cong 0,4 \omega_{2}$ allows to reduce resonant amplitude the more considerably, the higher is a shaft eigenfrequency (at a constant speed of rotor acceleration).

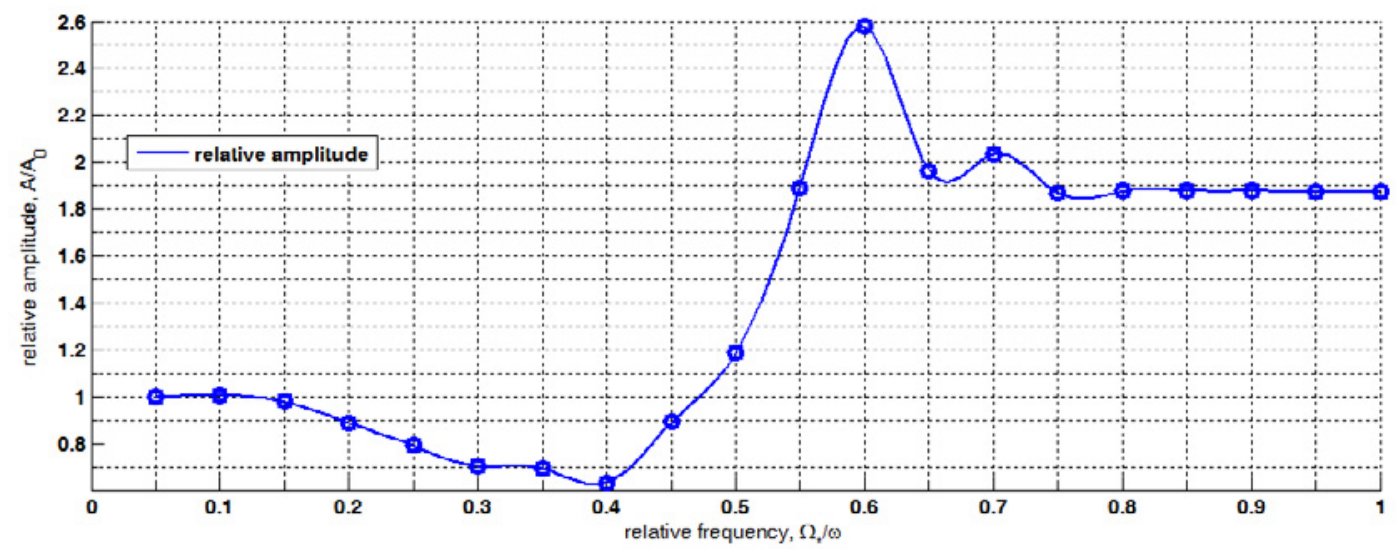

Fig.9 - Dependence of resonant oscillations amplitude on the moment of support stiffness change $\left(\omega_{2}=1\right)$ 


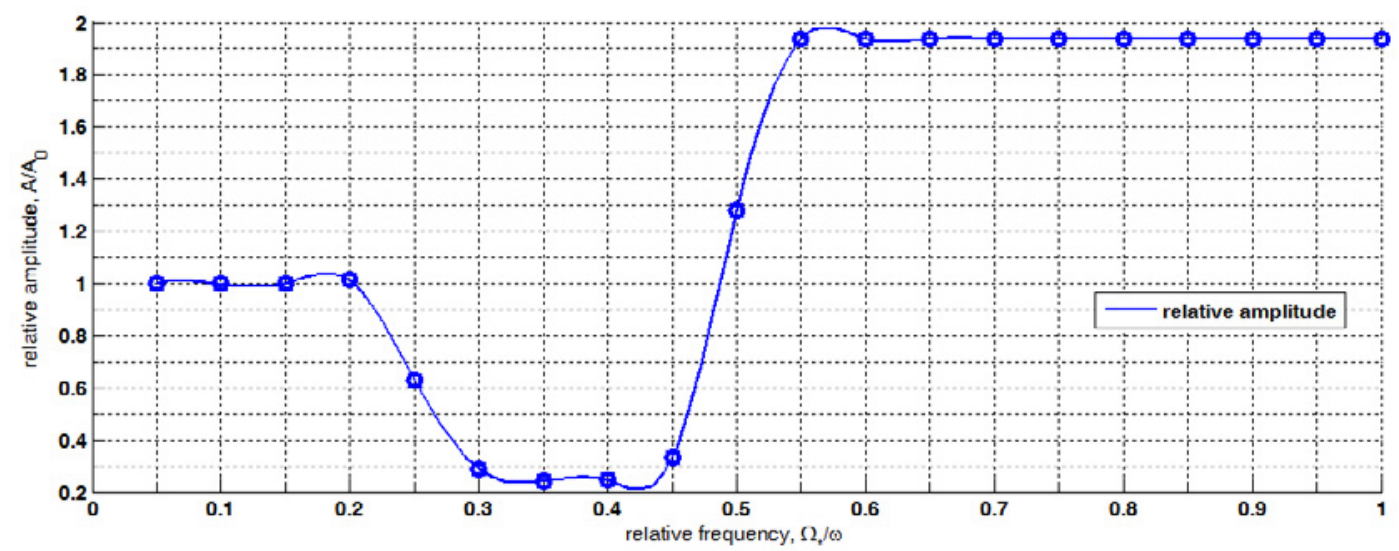

Fig.10 - Dependence of resonant oscillations amplitude on the moment of support stiffness change $\left(\omega_{2}=5\right)$

The results confirm efficiency of the offered method of transition through a resonance: resonant amplitude is reduced in times in comparison with a case when working stiffness joins at the beginning of rotor acceleration.

The offered algorithm of transition through critical frequency in some sense differs (but it can be compatible) from a method of operated transition through the critical frequency [6] which is based on a construction of engine acceleration control algorithm, optimal by criterion of the minimum time.

\section{References}

1. V. F. Zhuravlev, D. M. Klimov. Applied methods in theory of ocsillations (Nauka, Moscow, 1988) (in Russian)

2. Ali. H. Nayfeh. Introduction to Perturbation Techniques(John Wiley \& Sons, Inc, Canada, 1981)

3. Bogolyubov N. N., Mitropolsky Yu.A. Asymptotic methods in theory of nonlinear oscillations (Nauka, Moscow, 1974) (in Russian)

4. Urabe M. Galerkin method for nonlinear periodic systems, Mechanic, 97 (1996)

5. Merkin M.R., Friedman V.

M.Projectivemethodforforcednon-stationary oscillations in nonlinear systems with slowly varying parameters, Applied Mathematics and Mechanics, 45 (1981) (in Russian)

6. Malinin L.M. Pervozvansky A.A. Optimization of the unbalanced rotor transition through a critical velocity, Engineering Science, 4(1983) (in Russian)

7. Pechenev A.V. About movement of oscillatory system with limited excitement near a resonance, DASUSSR, 290(1986) (in Russian)
Subject of further research is creation of approximate analytical solutions in case of fast magnets drawing apart (deviation) and non-stationary excitement frequency, and also performance of calculations with the clarified elastic characteristic of a magnetic support. Such a research is necessary for the purpose of development of the most acceptable criterion of rotor acceleration with critical frequency overcoming. 